\title{
Tumors associated with oncogenic viruses and recombinant vaccines
}

\begin{abstract}
Oncogenic viruses are responsible by development of tumors as Human papillomavirus (HPV) that infect the anogenital epithelium causing cervical cancer, anal and penis cancer. There are viral groups based on their oncogenic activity as high-risk types, low-risk types and types of undetermined-risk. Several biotechnological assays are based on the production of recombinant products originated by the interaction of a viral genome with the genome of a host cell as bacteria, yeast, cell cultures and insects. Recently have been used virus-like particles (VLP) independently of the viral genome since some viral particles have the ability to self-assembling. There are some approved VLP vaccines as HPV vaccines, Hepatitis B and E vaccines and other VLP based vaccines under development such as using Human B19 parvovirus-like particles, Influenza vaccine candidates, Norwalk virus, Ebola and Marbug virus, Hepatitis C, Human immunodeficiency virus (HIV) as vaccine candidates. The aim of this present study was to address some examples of virus causing tumors and their respective recombinant protein based virus-like particle vaccines. Besides of HPV, also will also be addressed Hepatitis C virus (HCV), Hepatitis B virus (HBV), Herpesvirus (HSV), Herpes Simplex Virus (HSV-1) and Herpes Simplex Virus (HSV-2), Human Herpesvirus 3 (HHV-3), Human Herpesvirus 4 (HHV-4) or Epstain-Barr virus (EBV), Human Herpesvirus-5 (HHV-5) or Human Cytomegalovirus (HCMV), Human Herpesvirus 6 (HHV-6), Human Herpesvirus 7 (HHV-7) and Human Herpesvirus 8 (HHV-8), also known as Kaposi's sarcoma-associated herpesvirus (KSHV). There are several challenges in the production of the virus-like particles based recombinant vaccines under development using specific cell lines and assays with laboratory animals. It is important to reflect about on the extent to which the pharmaceutical industries are willing to develop recombinant vaccines in the prevention of tumor associated diseases
\end{abstract}

Keywords: diseases, oncogenic viruses, recombinant vaccines, tumors
Volume 5 Issue 3 - 2018

\section{Dra Rachel Siqueira de Queiroz Simões, Dra Ortrud Monika Barth}

Laboratory of Morphology and Viral Morphogenesis, Instituto Oswaldo Cruz, Brazil

\author{
Correspondence: Dra Rachel Siqueira de Queiroz Simões, \\ Laboratory of Morphology and Viral Morphogenesis, Instituto \\ Oswaldo Cruz, Fundação Oswaldo Cruz, Avenida Brasil 4365, \\ 21040-900, Rio de Janeiro, Brazil, \\ Email rachelsqsimoes@gmail.com
}

Received: April 20, 2018 | Published: May 16, 2018

\begin{abstract}
Abbreviations: HPV, human papillomavirus; VLP, viruslike particles; HIV, human immunodeficiency virus; HCV, hepatitis C virus; HSV, herpesvirus; HSV-1, herpes simplex virus; HSV-2, herpes simplex virus; HHV-3, human herpesvirus 3; HHV-4, human herpesvirus 4; EBV, epstain-barr virus; HHV-5, human herpesvirus-5; HCMV, human cytomegalovirus; HHV-6, human herpesvirus 6; HHV-7, human herpesvirus 7; HHV-8, human herpesvirus 8; KSHV, kaposi's sarcoma-associated herpesvirus; MCD, multicentric castleman's disease
\end{abstract}

\section{Introduction}

Several viruses are causing tumors and are called oncogenic viruses. Once introduced into an organism, the strategies taken are virus-specific, reaching organs predestined to the development of tumors. Vaccines, of variable efficacy have been developed over the years, making use of the most diverse biotechnological production. The most recent are based on the production of recombinant products originated by the interaction of a viral genome with the genome of a host cell (bacteria, yeast, cell cultures, insects, etc.), which will express the desired proteins for immunization in case of infection by an infectious viral agent. Some viral particles have the ability to self-assemble into virus-like particles (VLP) independently of the viral genome. There are some approved VLP vaccines as Human papillomavirus (HPV) vaccines, Hepatitis B and E vaccines. ${ }^{1}$ There are other VLP based vaccines under development such as using Human B19 parvovirus-like particles, ${ }^{2}$ Influenza vaccine candidates, Norwalk virus, Ebola and Marbug virus, Hepatitis C, Human immunodeficiency virus (HIV) as vaccine candidates. ${ }^{1}$ However, the focus of the present scientific considerations address some examples of virus causing tumors and their respective recombinant protein based virus-like particle vaccines.

\section{Human papillomavirus (HPV)}

Papillomaviruses are non-enveloped viruses presenting a closed circular double-stranded non-segmented DNA genome of approximately $8 \mathrm{~kb}$. HPV induce the most common sexually transmitted disease and has been classified in the Alphapapillomavirus genus. ${ }^{3,4}$ Detection of viral DNA sequences of the L1 gene, the stable and most conserved region of the viral genome, has been generally performed. More than $200 \mathrm{HPV}$ genotypes and several HPV types associated with particular diseases as oral lesions (Hecks disease, oropharyngeal carcinoma, laryngeal papillomas), anogenital warts (Bowenoid papulosis, Buschike-Lowenstein tumor), Epidermodysplasia verruciformis (plane warts, Pityriasislike plaques, squamous cell carcinomas of sun-exposed skin) have been described. ${ }^{5,6} \mathrm{HPV}$ vaccination is the best preventive strategy against cervical cancer, cervical intraepithelial neoplasia and genital warts. A recombinant DNA technology has allowed the development 
of prophylactic vaccines for HPV. Licensed HPV recombinant vaccines, as bivalent (Cervarix $\left.{ }^{\circledR}\right)$ against HPV16/18, quadrivalent (Gardasil $\left.{ }^{\circledR}\right)$ target HPV16/18/6/11 and nonavalent (Gardasil-9®) target HPV16/18/31/33/45/52/58/6/11 have been applied in many countries. ${ }^{4,5}$ Baculovirus and yeast (Saccharomyces cerevisiae) were the target as an expression system. Papillomaviruses can be used as viral vectors in the gene therapy and new therapeutic targets have been successfully applied by recombinant DNA methods. ${ }^{1,5}$

The Human papillomavirus (HPV) is critical in the pathogenesis of cervical cancer, the most common cancer among women. The natural history and epidemiologic studies of HPV are key tools for possible changes in cervical screening and vaccine immunization strategies. Several studies discuss the molecular detection of viral DNA sequences in recombinant DNA products and the interaction of viral chromatin with the host cell chromatin during viral replication. One of the research approaches investigates morphological alterations of HPV-positive human cervical carcinoma cell lines ( $\mathrm{SiHa}$ and $\mathrm{HeLa}$ ) as possible prognostic markers of cervical cancer. Gene therapy has a great potential as a new therapeutical modality in keratinocyte reprogramming, which can be a crucial point for the study of HPV tumors associated in regenerative medicine at the tissue engineering laboratories. New biotechnologies in molecular biology have been applied in research of HPV and are being investigated in gene therapy. ${ }^{1,4,6}$

\section{Hepatitis C virus (HCV)}

Hepatitis $\mathrm{C}$ virus (HCV) belongs to the Nidovirales order, Flaviridae family, Hepacivirus genus. HCV is an enveloped virus presenting a single strand of positive polarized RNA genome with approximately 9,400 nucleotides. HCV illness is a chronic infection that affects more than $2 \%$ of the global population and causes endstage liver diseases as chronic hepatitis. It is a worldwide public health problem that affects more than $70 \%$ of the estimated 170 million people inducing chronic lesions hepatitis. This virus leads to severe fibrosis and cirrhosis, hepatic failure, or hepatocellular carcinoma. HCV has a higher rate of mutation existing inside an individual as quasispecies. ${ }^{7} \mathrm{HCV}$ is divided into six genotypes and multiple subtypes. ${ }^{8}$ The envelope glycoproteins E1 and E2 are the natural targets to neutralizing antibodies response, but are also the two of the most variable HCV proteins. Production of specific antibodies in rabbits against conserved and potentially immunogenic peptides of the HCV envelope glycoprotein E2 has been described.

$\mathrm{HCV}$ displays a high variability and is classified into seven genetically distinct genotypes which differ by approximately $30 \%$ at the nucleotide level. Envelope (E1/E2) proteins of HCV may generate neutralizing antibodies. At the end $\mathrm{N}$-terminus of the $\mathrm{E} 2$ protein there is a region of 27 amino acids called hypervariable region 1 (HVR1), very important in neutralizing HCV. Despite the high degree of variability of E2 protein, some amino acid positions are conserved and this protein is the target of several neutralizing monoclonal antibodies. ${ }^{8-11}$ More biotechnological studies are need to investigate the clinical and epidemiological aspects and to associate the presence of antibodies with the progression of liver diseases. ${ }^{1,12}$ However, the high variability of this antigenic fragment plays a key role in the viral escape mechanism of the host immune response and represents the major obstacle in the development of an HCV vaccine. ${ }^{13}$
New biotechnologies in molecular biology as quimeric vaccine production using conserved peptides are possible candidates of peptide vaccine against HCV infection. ${ }^{10}$ Some studies involved the detection of antibodies in rabbits with immunogenic potentially activity by synthetic peptides of the HCV envelope glycoprotein E2 in chronic $\mathrm{HCV}$ infections. ${ }^{11}$ Until the date $\mathrm{HCV}$ vaccine has not been available.

\section{Hepatitis B virus (HBV)}

Hepatitis B virus (HBV) is classified in the Hepadnaviridae family. This family is divided into two genera: Orthohepadnavirus and Avihepadnavirus. The genome is composed of a 3,200base pairs (bp) of a circular double stranded DNA presenting four open reading frames (ORFs) designated as: Pre-S/S, Pre C/C, P and X. The Pre-S/S ORF corresponds to the Hepatitis virus surface gene (HBsAg). The Pre-S1, Pre-S2 and S regions have three initiation codons in the same reading phase that, after being translated, originate the proteins: $\mathrm{L}$ "Large" (400aa), Middle "M" (281aa) and "Small" (226aa). These three proteins that comprise the HBsAg are found in the serum of infected individuals in two ways: Small glycosylated (GP27) and Small non-glycosylated (P24), Middle glycosylated (GP33) and Middle di-glycosylated (GP36), and Large glycosylated (GP42) and Large non-glycosylated L (P39). ${ }^{14}$

$\mathrm{HBV}$ is one of the major causative agents of chronic liver disease. It is estimated that about 350 million people worldwide are carriers of the HBV and approximately 2 million are infected individuals in Brazil. The first reports of variability of the Hepatitis B virus (HBV) were described by Le Bouvier (1971), who identified two mutually exclusive antigenic determinants, $d$ and $y$, located in HBsAg. Two additional determinants, $w$ and $r$, were subsequently enunciated by Bancroft et al. (1972). ${ }^{14}$ Thus, nine subtypes have been described: ayw1, ayw2, ayw3, ayw4, adw2, adw4, ayr, adrq + and adrq-, which share a common conformational epitope present in HBsAg. There are eight $\mathrm{HBV}$ genotypes (identified as $\mathrm{A}-\mathrm{H}$ ) that exhibit more than $8 \%$ divergence between complete genomic sequences. The analysis of genomic variability of $\mathrm{HBV}$ isolates is fundamental for molecular and epidemiological studies. ${ }^{14,15}$

HBV infection produces two types of viral particles: full, spherical, HBV genome-containing infectious particles $(42 \mathrm{~nm})$, as well as non-infectious spherical or filamentous $(22 \mathrm{~nm})$ subviral particles composed exclusively of HBsAg. An expression system (as used in the Papilloma vaccines, the first recombinant vaccine licensed and produced from yeast expression) was used for Hepatitis B surfaceantigen isolation from human plasma of chronic HBV patients (Heptavax-B, Merck \& Co), and was released in 1981. ${ }^{16}$

Worldwide over the last 30years, HBsAg has been used as a commercial vaccine against hepatitis B. Actually, there are two types of successful vaccines based on virus-like particles (VLP) involving Hepatitis B virus surface antigen ( $\mathrm{HBsAg}$ ) and core antigen ( $\mathrm{HBcAg}$ ) expressed in Escherichia coli. ${ }^{1,17}$ Conditions on the immunogenicity can be tested in mice with alternative routes of administration of HBV vaccine and novel formulations assays. ${ }^{1}$

The development of recombinant vaccines composed exclusively of HBsAg (Engerix-B, SmithKline and Recombivax, Merck \& Co) was possible with the advance of genetic engineering. New 
researches have investigated the improvement of the vaccine model of heterological antigens based on hepatitis B virus envelope protein containing $\mathrm{HCV}$ antigen as a quimeric vaccine.

\section{Herpesvirus (HSV)}

Another oncogenic virus is the Herpesvirus (HSV) which viral replication sites occur inside the cell nucleus. HSV can infect and establish latent infections as in mice, guinea pigs, rabbits and humans. The viral genome in a latent state (episomal) has been described inside the nucleus of sensory ganglions.

\section{Herpes simplex virus (HSV-I) and herpes simplex virus (HSV-2)}

Herpes simplex virus (HSV-1) or Human herpesvirus 1 (HHV-1) induce an acute infection accompanied by the formation of labial vesicles in the oral region. Herpes simplex virus (HSV-2) or Human herpesvirus 2 (HHV-2) are causing strong lesions in the genital area. These herpesviruses are members of the Alphaherpesviruses subfamily. There are no licensed vaccines against HSV-1 and HSV $-2,{ }^{18}$ despite there are preventive and therapeutic vaccines for HSV-2, specially of various stages of development in clinical testing. ${ }^{19}$

\section{Human herpesvirus 3 (HHV-3)}

Human herpesvirus 3 (HHV-3) or varicella-zoster virus is highly contagious and has approximately 69 different genes being five homologous genes of HSV. It is classified in the Alphaherpesvirinae subfamily and the Varicellovirus genus with DNA of $125 \mathrm{kbp}$, which is lower than the HSV (152kbp). Primary varicella-zoster virus infection causes varicella (chickenpox) and when occurs the reactivation of latent virus, usually causes herpes zoster in adults manifesting as vesicular rash. The Advisory Committee on Immunization Practices and Center for Diseases Control and Prevention (CDC) recommends for Herpes Zoster prevention a single dose of Zostavax ${ }^{\circledR}$ (live attenuated virus vaccine) for people 60 years old or older. This vaccine is not indicated for the treatment of zoster or postherpetic neuralgia $(\mathrm{PHN})$ and is not indicated for prevention of primary varicella infection (Chickenpox). ${ }^{20}$

\section{Human herpesvirus 4 (HHV-4)}

Human Herpesvirus 4 (HHV-4) or Epstain-Barr virus (EBV), a member of the Gammaherpesvirus subfamily, was discovered in 1964 by Tony Epstein and Yvone Barr in tumor cells of patients presenting Burkitt's lymphoma. There are three latency forms: Lat I, LatII and Lat III. The Lat I site express the single protein EBNA 1 (EpsteinBarr nuclear antigen) and express two sites of RNA (Epstein-Barr early RNA-EBERS) and (Bam A). The Lat II site express additional proteins (latent membrane protein - LMPs) and Lat III express more proteins as EBNAs 2, 3A, 3B, 3C and LP. There are several types of tumors associated with Epstein-Barr virus (EBV) such as Burkitt's lymphoma, Hodgkin's disease, T-Cell lymphoma, post-transplant lymphoproliferative disease, nasopharyngeal carcinoma, gastric carcinoma and Leiomyosarcoma. ${ }^{21}$ EBV peptide vaccine has been tested in the rabbit model using immunogenic epitopes selected to stimulate both the T as well as B lymphocytes. ${ }^{18}$

\section{Human herpesvirus-5 (HHV-5)}

Human Herpesvirus-5 (HHV-5) or Human Cytomegalovirus
(HCMV) belong to the Herpesviridae family, Betaherpesviridae subfamily, Cytomegalovirus genus. They are frequently detected in transplanted patients and immunocompetent individuals, where they mediate early (IE) proteins that are responsible for the regulation of the early (E) proteins involved in cell regulation including the expression and transport of the HLA antigens (class I MHC proteins) to the proteasome. In particular, the phosphoprotein pp65 can be used as early markers of the virus infection in cell cultures..$^{22}$ The reactivation in transplantation patients has been performed by quantitative HCMV DNA detection from whole blood or plasma and quantitative detection of pp65 antigen in neutrophil granulocytes. ${ }^{22}$ Antiviral agents, that are currently available, are used for prevention and therapeutics of HCMV. Clinical assays for the development of vaccines don't have been successful due to the latent infection cycle of the virus and are not yet available. ${ }^{22}$

\section{Human herpesvirus 6 (HHV-6) and human herpesvirus 7 (HHV-7)}

Human herpesvirus 6 (HHV-6) induces severe cutaneous eruptions and roseola. Human herpesvirus 7 (HHV-7) has been detected in patients with pityriasisrosea, a common papulosquamous disease. ${ }^{23}$ Both viruses are closely related $\beta$-herperviruses and classified in the Herpesviridae family, Betaherpesvirinae subfamily, Roseolovirus genus. The genome of HHV-6 is of a linear double-stranded DNA with 159 to $170 \mathrm{kbp}$ containing approximately 100 genes, while the HHV-7 genome has only $133 \mathrm{kbp}$. The primary infection cause subit exanthema. The viruses are secreted by saliva in healthy populations and viral reactivation occurs in immuno-compromised individuals (AIDs patients stressed and transplanted patients). HHV-6 was isolated from the peripheral blood lymphocytes of patients with lymphoproliferative disorders and it was initially found within B cells of infected individuals. Posteriorly, CD4+ T cells were the major cell type infected by HHV-6. In 1991, researchers discovered two variants of HHV-6 designed HHV-6A and HHV-6B with $90 \%$ of genetic similarity. Both variants of HHV-6 A, B and HHV -7 presenting with $20 \%-75 \%$ nucleic acid homology depending on the genes. ${ }^{23}$ There are no commercially available recombinant vaccines for the treatment of HHV -6 and 7, only therapeutic measures with the administration of antiviral agents as for example in patients who have undergone bone marrow transplantation. However, mutations in homologous genes such as the HHV-6 U69 gene that is homologous to the HCMV UL97 gene is associated with decreased efficacy of ganciclovir.

\section{Human herpesvirus 8 (HHV-8)}

The Human Herpesvirus 8 (HHV-8), also known as Kaposi's sarcoma-associated herpesvirus (KSHV), is classified in the order Herspesvirales, Herpesviridae family, Gammaherpesvirinae subfamily and Rhadinovirus genus. The DNA viral genome is composed of a $145 \mathrm{kbp}$ region containing all open reading frame sequences. There are HHV8-exclusive ORFs designated K (ORF $\mathrm{K} 1-\mathrm{K} 15)$ being possible by sequencing hypervariable regions of the viral genome, such as ORF-K1 to identify 5 viral subtypes (A-E). HHV -8 is associated with Kaposi's Sarcoma (KS) of HIV positive and negative patients, and of two rare lymphoproliferative disorders: the Primary Effusion Lymphomas (PELs), called also Body-cavitybased lymphoma (it is a B cells lymphoma not the Hodgkin tumor), and the Multicentric Castleman's disease (MCD). DNA vaccines are of circular double-stranded plasmid DNA (pDNA) molecules and 
have been applied in the development of therapeutic or prophylactic vaccination for herpesvirus infections. ${ }^{21}$

\section{Conclusion}

In recent years, interest in the development of virus-like particles (VLP) has increased exponentially in the biomedical area as potential vaccine candidates and for application in gene therapy. ${ }^{2}$ Independent of the viral genome, VLPs are non-infectious bionanoparticles composed of structural proteins capable of self-assembly in viral capsid mimicking epitopes of antigens presented by tumor cells or external pathogens. ${ }^{2}$ VLPs are characterized using the combination of biochemical methods in the identification of structural proteins (Western Blot or mass spectrometry), biophysical methods used to measure the morphology and size of viral particles (transmission electron microscopy-MET) and chromatographic methods. There is a lot of technological challenges in the development of the therapeutic proteins for a high production in the biopharmaceutical sector, integrating upstream and downstream processing in bioreactors. ${ }^{1}$ Thus transient expression of recombinant plasmids transfected into several cell lines has been evaluated by immunobiological assays. ${ }^{24}$ Infectious diseases and their specific interactions with individual variability have provided an advance in clinical innovations as therapeutic targets, biomarkers and prevention, stemming from genetics variations (point mutations) and personalized medicine. The biotechnological research and development of a biopharmaceutical product as a drug needs a number of volunteers in clinical trials phase I, II and III until will be approved by the FDA, this can take at least 10 years on average. There are several challenges in the production of the virus-like particles based recombinant vaccines under development using specific cell lines and assays with laboratory animals. It is important to reflect about on the extent to which the pharmaceutical industries are willing to develop recombinant vaccines in the prevention of tumour associated diseases

\section{Acknowledgements}

This research was supported by the Coordenação de Aperfeiçoamento de Pessoal de Nível Superior (CAPES)/“Brasil Sem Miséria" Program and the Conselho Nacional de Desenvolvimento Científico e Tecnológico (CNPq).

\section{Conflict of interest}

The authors declare that they have no conflict of interest.

\section{References}

1. Jain NK, Sahni N, Kumru OS, et al. Formulation and stabilization of recombinant protein based virus-like particle vaccines. Adv Drug Deliv Rev. 2015;93:42-55.

2. Ladd Effio C, Wenger L, Ötes O, et al. Downstream processing of viruslike particles: Single-stage and multi-stage aqueous two-phase extraction. J Chromatogr A. 2015;1383:35-46.

3. Barth OM, Schatzmayr HG. Classificação e características gerais dos vírus patogênicos para o homem. In: Coura JR, editor. Dinâmica das Doenças Infecciosas e Parasitárias. Segunda edição, capítulo 148, Editora Guanabara Koogan, Brazil: Rio de Janeiro; 2013. p. 1680-1694.

4. Simões RSQ, Silva EP, Barth OM. Prevalence of High-Risk Human papillomavirus Genotypes and Predictors factors for Cervical Cancer in Unimmunized Brazilian Women without Cytological Abnormalities. Adv Biotech \& Micro. 2018;8(5):555749.
5. Simões RSQ, Barth OM. Papillomavirus: Viral vectors in the gene therapy and new therapeutic targets. International Journal of Biomedical Research. 2017;6(10):763-768.

6. Simões RSQ, Barth OM. Historical and Epidemiological Aspects of Some Human Diseases Just to Zika Virus, a Short Review. International Journal of Research Studies in Biosciences (IJRSB). 2016;4(4):46-54.

7. Martell M, Esteban JI, Quer J, et al. Hepatitis C virus (HCV) circulates as a population of different but closely related genomes: quasispecies nature of HCV genome distribution. $J$ Virol. 1992;66(5):3225-3229.

8. Bukh J, Emerson SU, Purcell RH. Genetic heterogeneity of hepatitis C virus and related viruses. In: Rizzeto M, Purcell RH, Gerin JL, Verme G, editors. Viral hepatitis and liver disease. Turin: Minerva Médica; 1997. p. $167-175$.

9. Drazan KE. Molecular biology of hepatitis C infection. Liver Transpl. 2000;6(4):396-406.

10. Marins RSQS, Mazzine RBO, Mouta SSJ, et al. Transient transfections of human cell lines HEK-293T and HUh7 for production of $\mathrm{HBsAg} / \mathrm{HCV}$ Chimeric Protein: comparative detection using the Elisa and Western Blotting. Virus Reviews and Research-Journal of the Brazilian Society for Virology. 2011;16:111-112.

11. Marins RSQS, Almeida EC, Freitas LJ, et al. Production of specific antibodies in rabbits against conserved and potentially immunogenic peptides of the HCV envelope glycoprotein E2. Virus Reviews and Research-Journal of the Brazilian Society for Virology. 2012;17:79.

12. Soares CC, Niel CMG, Mello FCA, et al. Hepatites virais. In: Santos NSO, Romanos MTV, Wigg MD, editors. Virologia Humana. Gen: Rio de Janeiro; 2015. p. 398-428.

13. Forns X, Bukh J, Purcell RH. The challenge of developing a vaccine against hepatitis C virus. J Hepatol. 2002;37(5):684-695.

14. Dehesa-Violante M, Nuñez-Nateras R. Epidemiology of hepatitis virus B and C. Arch Med Res. 2007;38(6):606-611.

15. Jin J, Yang JY, Liu J, et al. DNA immunization with fusion genes encoding different regions of hepatitis $\mathrm{C}$ virus $\mathrm{E} 2$ fused to the gene for hepatitis $\mathrm{B}$ surface antigen elicits immune responses to both HCV and HBV. World $J$ Gastroenterol. 2002;8(3):505-510.

16. Assad S, Francis A. Over a decade of experience with a yeast recombinant hepatitis B vaccine. Vaccine. 2000;18(1-2):57-67.

17. Yang Y, Li H, Li Z, et al. Size-exclusion HPLC provides a simple, rapid, and versatile alternative method for quality control of vaccines by characterizing the assembly of antigens. Vaccine. 2015;33(9):1143-1150.

18. Rajčáni J, Bánáti F, Szenthe K, et al. The potential of currently unavailable herpes virus vaccines. Expert Rev Vaccines. 2018;17(3):239-248.

19. Johnston C, Gottlieb SL, WaldA. Status of vaccine research and development of vaccines for herpes simplex virus. Vaccine. 2016;34(26):2948-2952.

20. Center for Diseases Control and Prevention-CDC; 2018.

21. Thomas G Evans, Mary Wloch. DNA vaccines for human herpesviruses. Human Herpesviruses: Biology, Therapy, and Immunoprophylaxis. USA: Vical Incorporated; 2007.

22. Schottstedt V, Blümel J, Burger R, et al. Human Cytomegalovirus (HCMV)-Revised. Transfus Med Hemother. 2010;37(6):365-375.

23. Andrew Blauvelt. Skin Diseases Associated with Human Herpesvirus 6, 7, and 8 infection. Journal of Investigative Dermatology Symposium Proceedings. 2001;6(3):197-202.

24. Steppert P, Burgstaller D, Klausberger M, et al. Quantification and characterization of virus-like particles by size-exclusion chromatography and nanoparticle tracking analysis. J Chromatogr A. 2017;1487:89-99. 